\title{
Magnetic Relaxation and Memory Effect in Nickel-Chromium Cyanide Nanoparticles
}

\author{
A. Zentko, V. KaveČanský, M. Mihalik, S. MaŤaš, \\ Z. Mitróová, M. Zentková
}

Institute of Experimental Physics, Slovak Academy of Sciences

Watsonova 47, 04001 Košice, Slovak Republic

M. MARYŠKO

Institute of Physics of the AS CR, v.v.i.

Na Slovance 2, 18221 Prague 8, Czech Republic

\section{AND Z. JAGLIČIĆ}

Institute of Mathematics, Physics and Mechanics

Jadranska 19, 1000 Ljubljana, Slovenia

\begin{abstract}
The low temperature dynamics of a magnetic nanoparticle system $\mathrm{Ni}_{3}\left[\mathrm{Cr}(\mathrm{CN})_{6}\right]_{2}$ with an average nanoparticles size of $4 \mathrm{~nm}$ was studied. Using different temperature and field protocols memory phenomena were studied by the DC magnetization and magnetic relaxation measurements of the system at temperatures below $T_{\mathrm{m}}=19 \mathrm{~K}$. Aging experiments show an absence of any waiting time dependence in the magnetization relaxation due to a field change after zero field and field cooling. This observation discriminates the dynamics of the system from the behaviour of a classical spin-glass.
\end{abstract}

PACS numbers: 75.50.Tt, 75.50.Lk

\section{Introduction}

In recent years the dynamics of magnetic nanoparticle (NAP) systems has been the subject of considerable attention, partly for the potential applications in the field of high density recording media, but also for exotic behaviour such as macroscopic tunnelling [1] and quantum computing [2]. Because of the single domain nature of the magnetic NAPs, they are usually modelled as giant spin 
objects with significant anisotropy. The magnetic dynamics of single domain particles is simpler than those of bulk magnets. The energy of single domain particle depends only on the direction of the magnetization vector. If there is any magnetocrystalline anisotropy in the particle, the two stable states "up" and "down" are separated by a potential barrier $\Delta E$. This results in the generation of a hysteresis loop in the magnetization curve. However, the NAP moments thermally fluctuate between their easy directions and with decreasing temperature freeze along the easy direction at the blocking temperature $T_{\mathrm{B}}$. The relaxation rate of relaxation process $1 / \tau$ is governed by the Arrhenius thermal activation law. The rate is proportional to $\exp \left(-\Delta E / k_{\mathrm{B}} T\right)$. We present our results on relaxation process and memory effect in the $\mathrm{Ni}_{3}\left[\mathrm{Cr}(\mathrm{CN})_{6}\right]_{2}-\mathrm{NAP}$ system. The magnetic NAP sample was prepared using reverse micelles techniques [3]. The X-ray powder diffraction pattern on this sample (Fig. 1a), taken by Philips X-Pert diffractometer at $\mathrm{Cu} K_{\alpha}$ radiation, revealed that the average NAP size is $4 \mathrm{~nm}$. The magnetic measurements were carried out on a SQUID magnetometer (MPMS) in the temperature range from 2 up to $100 \mathrm{~K}$. The number of water molecules in NAP and the content of organic surfactant were determined by thermo-gravimetric measurements (SETARAM TG/DTA 9L).

\section{Results and discussion}

Figure $1 \mathrm{~b}$ displays the temperature dependence of the magnetization $M(T)$ for the typical sample of Ni-NAPs taken in zero field cooled (ZFC) and field cooled (FC) regime. The curves exhibit the typical characteristics of a superparamagnetic system, i.e., ZFC and FC curves follow a Curie-Weiss like paramagnetic behaviour
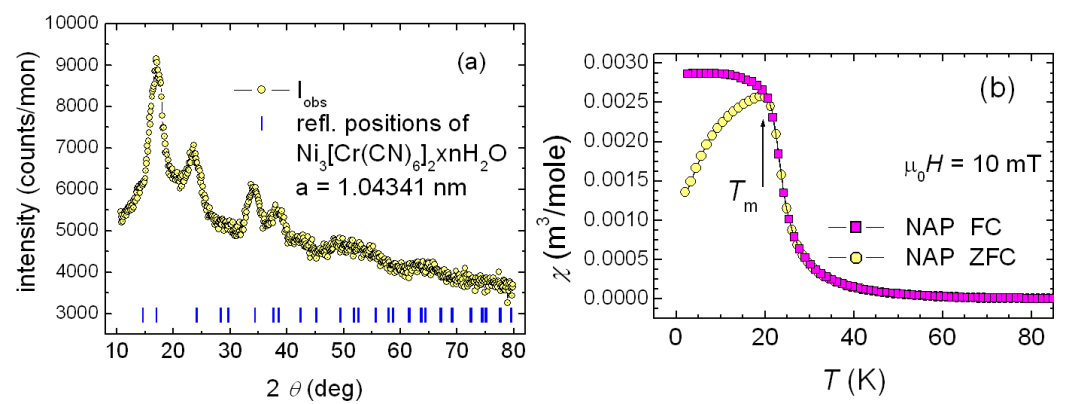

Fig. 1. X-ray diffraction pattern shows relatively broad peaks, reflecting small particle sizes (a); ZFC and FC magnetization measurement (b).

at higher temperatures with parameters $\theta=64 \mathrm{~K}$ and $C=3.2 \mathrm{emu} \mathrm{K} / \mathrm{mol} . M(T)$ rapidly increases below $25 \mathrm{~K}$ as temperature is decreased. As $T$ is further lowered $M(T)$ exhibits strong irreversibility, given by the divergence of the $M_{\mathrm{fc}}(T)$ and $M_{\mathrm{zfc}}(T)$ curves below the bifurcation temperature $T_{\mathrm{irr}}$ at which $M_{\mathrm{zfc}}(T)$ starts to deviate from $M_{\mathrm{fc}}(T)$. $M_{\mathrm{zfc}}(T)$ exhibits a maximum at a field dependent temper- 

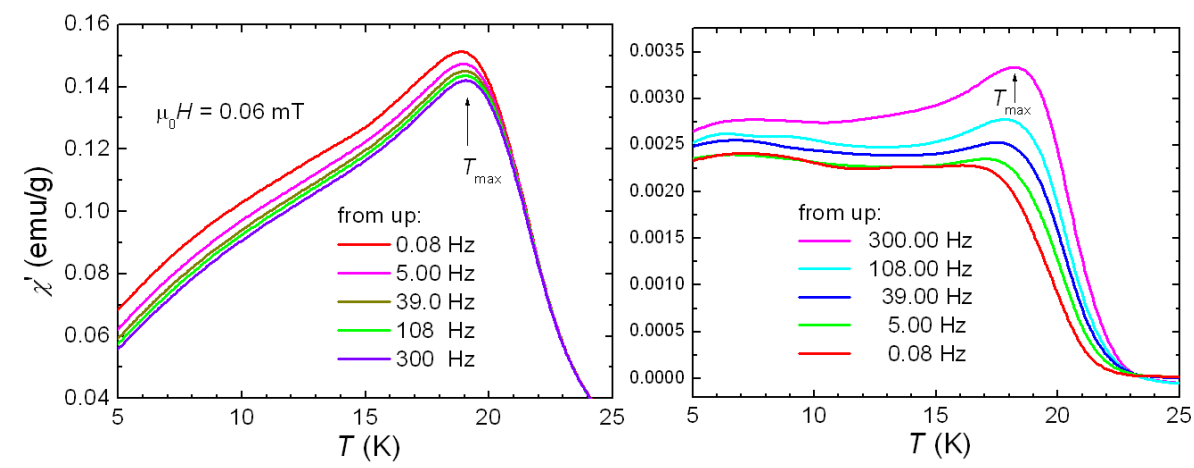

Fig. 2. AC susceptibility: in-phase $\chi^{\prime}(T)$ and out-phase $\chi^{\prime \prime}(T)$.

ature $T_{\mathrm{m}}<T_{\mathrm{irr}}\left(T_{\mathrm{m}}=19 \mathrm{~K}\right)$, while the $M_{\mathrm{fc}}(T)$ curve confirms an increase with decreasing temperature. The temperature $T_{\text {irr }}$ is shifted to lower temperatures as $H$ is increased.

The frequency dependence of the AC susceptibility is shown in Fig. 2. Both $\chi^{\prime}(T)$ and $\chi^{\prime \prime}(T)$ curves show frequency dependent peaks at $T_{\max }=19 \mathrm{~K}$ and $T_{\max }=18 \mathrm{~K}$ for $\chi^{\prime}(T)$ and $\chi^{\prime \prime}(T)$, respectively. The raising of the frequency tends to increase $T_{\max }$ and also $\chi^{\prime \prime}(T)$ values; on the other hand, it decreases $\chi^{\prime}(T)$ value. The rotation of the high-spin magnetic moment of the cluster is gradually frozen around $19 \mathrm{~K}$ owing to intra-cluster potential barrier and the out-phase component $\chi^{\prime \prime}$ arises. It is assumed that the relaxation rate $1 / \tau$ agreed with the frequency of the $\mathrm{AC}$ magnetic field, i.e. $1 / \tau=2 \pi f_{0}$ at the temperature of the maximum in $\chi^{\prime \prime}(T)$. By fitting the Arrhenius law $\tau=\tau_{0} \exp \left(\Delta E / k_{\mathrm{B}} T\right)$ we obtained: $\tau_{0}=6.4 \times 10^{-12} \mathrm{~s}$ and $\Delta E=151 \mathrm{~K}$. On the basis of these parameters, the blocking temperature $T_{\mathrm{B}}$, where the magnetic relaxation time of the clusters becomes longer than the time scale of the DC magnetization measurements $(\tau=100 \mathrm{~s})$, was calculated to be roughly $11.5 \mathrm{~K}$. This value corresponds to the temperature below which a hysteresis loop appears in magnetization curve. Since the bifurcation of the $M(T)$ curves occurs for both interacting (spin-glass like) and non-interacting (superparamagnetic) nanoparticle systems, the history-dependent behaviour of $\mathrm{Ni}_{3}\left[\mathrm{Cr}(\mathrm{CN})_{6}\right]_{2}-\mathrm{NAP}$ was also studied [4]. While the DC magnetic relaxation of a spin glass is known to be strongly age dependent, there is no evidence that superparamagnetic systems exhibit pronounced aging dependence. Our sample was cooled from high enough temperature (higher than the blocking temperature) to measuring temperature, in our case cooled from $100 \mathrm{~K}$ down to $5 \mathrm{~K}$ in zero magnetic field (or in 5, 10, 15 Oe), the field was applied (or cut off) after waiting times $\left(t_{\text {waiting }}=100,300,500 \mathrm{~s}\right)$ and the time evolution of magnetization is recorded.

The ZFC relaxation curves $M(t)$ displayed in Fig. 3a indicate the absence of any dependence on the waiting time, while the thermo-remanent magnetiza- 

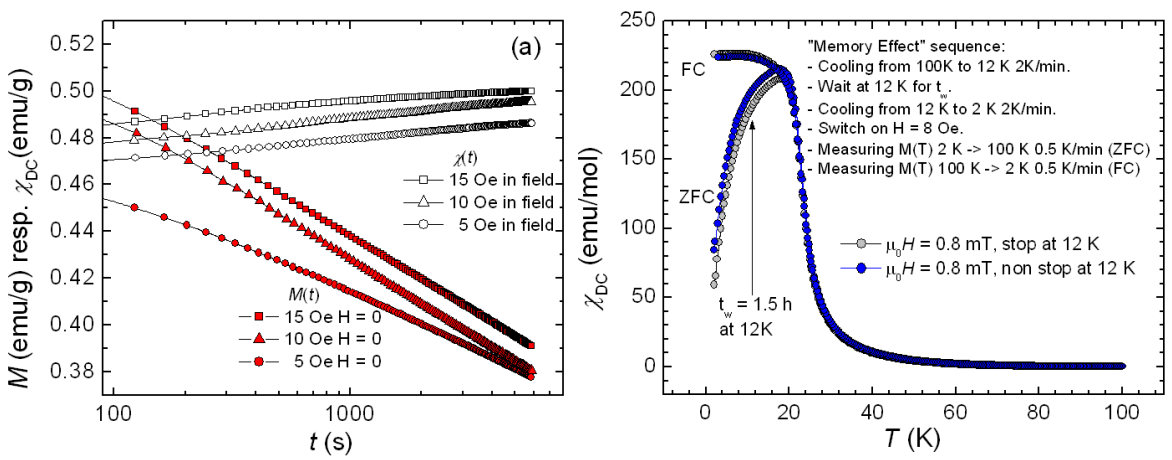

Fig. 3. Magnetic relaxation data (a); $M_{\mathrm{zfc}}(T)$ measured with and without a stop (b).

tion relaxation data $\chi_{\mathrm{DC}}(t)$ displayed in Fig. 3a indicate only a weak waiting time dependence. This suggests that the magnetic relaxation in this sample is predominantly controlled by thermally activated dynamics of individual particles rather than by interactions between magnetic NAPs typically associated with spin-glass behaviour. In order to get more information on the magnetic state of investigated sample the presence of the memory effect was probed. The ZFC curves were measured with a stop and without the stop (reference curve) during the cooling process. The measurements were performed under field $H=8$ Oe with and without a halt, $t_{\mathrm{w}}=5400 \mathrm{~s}$ at $T_{\mathrm{w}}=12 \mathrm{~K}$. The two ZFC curves $\left(\mathrm{ZFC}\right.$ and $\mathrm{ZFC}_{\text {ref }}-$ without a stop) together with FC curve are shown in Fig. 3b. The halted and reference curves do superimpose for all temperatures above $T_{\mathrm{m}}$. Below $T_{\mathrm{m}}$ the $\chi_{\mathrm{zfc}}(T)$ curves are under $\chi_{\mathrm{zfc}}(T)$ reference curve, however no anomaly dip has been observed in the vicinity of $T_{\mathrm{w}}$. In conclusion, we can say that the magnetic interactions between the clusters in $\mathrm{Ni}_{3}\left[\mathrm{Cr}(\mathrm{CN})_{6}\right]_{2}-\mathrm{NAP}$ system are negligibly weak, because of the long distance between neighbouring clusters. Therefore the properties described above certainly originate from single clusters.

\section{Acknowledgments}

This work was supported by projects APVT-20-005204, VEGA 2/7184/27 and ISTC SK-113/CZ-46.

\section{References}

[1] L. Thomas, F. Lionti, R. Ballou, D. Gatteschi, R. Sessoli,B. Barbara, Nature 383, 145 (1996).

[2] M.N. Leuenberger, D. Loos, Nature 410, 789 (2001).

[3] L. Catala, T. Gacoin, J.-P. Boilot, E. Riviere, C. Paulsen, E. Lhotel, T. Mallah, Adv. Mater. 15, 826 (2003).

[4] T. Jonsson, J. Mattsson, C. Dujurberg, F.A. Khan, P. Nordblad, P. Svedlindh, Phys. Rev. Lett. , 75 (1995). 